\title{
Rursus
}

Russus

Poiétique, réception et réécriture des textes antiques

$6 \mid 2011$

Relire, récrire, prolonger. Adaptations gréco-latines

\section{La violence dans l'Héraclès furieux d'Euripide. Lecture girardienne}

Jacqueline Assaël

\section{(2) OpenEdition}

Journals

Édition électronique

URL : http://journals.openedition.org/rursus/563

DOI : $10.4000 /$ rursus.563

ISSN : 1951-669X

Éditeur

Université Nice-Sophia Antipolis

Référence électronique

Jacqueline Assaël, «La violence dans l'Héraclès furieux d'Euripide. Lecture girardienne », Rursus [En ligne], 6 | 2011, mis en ligne le 09 février 2011, consulté le 30 avril 2019. URL : http:// journals.openedition.org/rursus/563; DOI : 10.4000/rursus.563

Ce document a été généré automatiquement le 30 avril 2019

Rursus 


\title{
La violence dans l'Héraclès furieux d'Euripide. Lecture girardienne
}

\author{
Jacqueline Assaël
}

1 Dans La violence et le sacré, puis dans Des choses cachées depuis la fondation du monde ${ }^{1}$, René Girard identifie le rite sacrificiel comme le fondement sur lequel l'équilibre des sociétés archaïques s'est universellement construit. En effet, selon lui, tous les comportements sont déterminés par une aspiration, appelée "désir mimétique ", qui fait violemment rechercher la captation des biens acquis par l'autre, considéré comme un double enviable. Or, cette tendance innée à une rivalité agressive risque de détruire les structures sociales. Avec une conscience plus ou moins maîtrisée des mécanismes qui entrent en jeu, à un moment ou à un autre de leur développement, les collectivités humaines mettent donc en place le rituel religieux du sacrifice pour interrompre le cycle des attaques et des vengeances sanglantes. Une victime, ayant quelquefois le statut de bouc émissaire, réconcilie alors les antagonistes autour de la célébration de son élimination purificatrice, et les rancœurs réciproques s'effacent, de sorte que la paix peut revenir.

Dans cette vision de l'histoire, le désordre des civilisations s'amplifie à deux moments différents : tout d'abord lors de la phase de violence pré-sacrificielle, puis, au moment où, en raison de l'évolution des mentalités, le fonctionnement du rituel se dégrade. En effet, lorsque son bien-fondé est remis en cause ou lorsque son efficacité n'apparaît plus, avant qu'un autre système de régulation de type judiciaire ne soit établi, les violences prolifèrent dans un complet dérèglement. Ce temps reçoit le nom de « crise sacrificielle ". Or, René Girard trouve notamment une illustration de ce genre de perturbation à travers une scène de l'Héraclès furieux d'Euripide, dans laquelle un sacrifice de purification échoue et entraîne une crise de démence au cours de laquelle le héros massacre sa femme et ses enfants ${ }^{2}$.

De fait, cette situation dramatique signale et suscite une réflexion sur le sens et la valeur de tels rites religieux. Cet épisode s'insère d'ailleurs dans la représentation d'un mythe entièrement reconstruit par le poète grec, de manière à analyser les mécanismes qui provoquent une contamination de la violence ou qui l'enrayent, au sein des rapports privés ainsi que dans le cadre des cités grecques et même dans l'univers des hommes et 
des dieux. Car, chez Euripide, Héraclès commence par effectuer le cycle des douze travaux pour racheter, en quelque sorte, un meurtre commis involontairement par Amphitryon, qu'il considère comme son père. Puis il retourne auprès des siens, mais il trouve sa famille en danger de mort, menacée par un usurpateur assassin. Le héros tue alors le tyran. Mais lorsqu'il s'apprête à accomplir un sacrifice pour se purifier du sang versé, cette cérémonie religieuse tourne mal. Héraclès devient fou et, dans son délire, il tue sa femme et ses enfants. Dans les versions traditionnelles du mythe, l'ordre séquentiel est inverse, de même que le système des causalités : l'extermination des monstres de la terre représente alors une expiation des actes sanglants commis par le héros lui-même ${ }^{3}$. Euripide réinterprète donc de manière originale l'enchaînement des phases de violence et la valeur des rituels sacrificiels. Or, parmi tous les éléments de cette mise en scène illustrant la réflexion d'Euripide, R. Girard choisit de commenter l'échec du sacrifice de purification qui lui semble signaler une remise en cause de ce type de pratique religieuse.

dans la lecture qu'il propose de cet épisode, tout se passe comme si, au lieu de se laver de sa souillure, Héraclès propageait une violence meurtrière au sein même de son propre foyer :

Le sacrifice projeté par le héros ne réussit que trop à polariser sur lui la violence. Celle-ci est simplement trop abondante, trop virulente. Le sang, comme le suggère Amphitryon, le sang versé dans de terribles travaux et en dernier lieu dans la cité elle-même, égare l'esprit d'Héraclès. Au lieu d'absorber la violence et de la dissiper au-dehors, le sacrifice ne l'attire sur la victime que pour la laisser déborder et se répandre de façon désastreuse aux alentours. Le sacrifice n'est plus apte à accomplir sa tâche; il vient grossir le torrent de la violence impure qu'il ne peut plus canaliser. Le mécanisme des substitutions s'affole et les créatures que le sacrifice devrait protéger deviennent ses victimes. ${ }^{4}$

R. Girard explique aussi l'échec du rituel par le fait que, d'un point de vue religieux, le meurtre de Lycos est d'une autre nature que les violences exercées dans le cadre des douze travaux :

il pourrait bien constituer un premier maillon de la violence impure 5 .

En effet, lorsque Héraclès prend en charge la faute meurtrière commise par Amphitryon à l'encontre d'Électryon, père d'Alcmène, il s'engage à purger la terre de ses monstres, à titre de compensation et d'expiation, par obéissance à des prescriptions divines ${ }^{6}$. Mais en tuant Lycos, le héros agit pour son propre compte. Dans cette perspective, le dysfonctionnement du sacrifice mis en scène par Euripide dénoncerait la banalisation d'une effusion de sang, c'est-à-dire la perte du sentiment du sacré attaché à la vie, l'inconscience profonde du sacrilège que représente le fait de tuer un être humain. Dans ce cas, d'ailleurs, les gestes de purification n'ont pas de véritable signification. En effet, les officiants ne respectent qu'un conformisme dont le poète souligne l'inanité. Il met en œuvre, à cette fin, des ressorts dramatiques marqués par une ironique perversité destructrice. D'après cette interprétation, Euripide exprimerait bien un point de vue né dans des temps de « crise sacrificielle », lorsque ces pratiques n'étant plus fondées sur un vif sens du sacré, leurs vertus sont finalement critiquées et mises en doute.

7 René Girard observe, en outre, que les tragiques grecs traitant du mythe d'Héraclès mettent en scène un tel "retournement catastrophique du sacrifice» de manière récurrente. Ainsi, dans les Trachiniennes de Sophocle, le héros allume un grand feu pour accomplir un rituel d'offrande et de purification, mais la chaleur de la flamme éveille alors la virulence du poison dont est enduite la tunique offerte par Déjanire. Dans sa douleur folle, Héraclès tue alors le messager Lichas. Pour R. Girard, le meurtre de ce 
serviteur, tout comme celui de l'usurpateur Lycos chez Euripide, déclenche un engrenage de violence impure ${ }^{7}$. Dans ces conditions, il semble effectivement que les poètes placent la figure d'Héraclès au centre d'une réflexion portant sur la possibilité de se purifier de la violence.

En réalité, l'épisode étudié par $\mathrm{R}$. Girard n'a pas seulement un intérêt ponctuel. Lui-même l'exploite uniquement pour donner un exemple du fonctionnement schématique de ce qu'il appelle la «crise sacrificielle» et il focalise son analyse sur cet événement particulier de l'intrigue :

Dans La folie d'Héraklès d'Euripide, il n'y a pas de conflit tragique, pas de débat entre des adversaires affrontés. Le sujet réel est l'échec d'un sacrifice, la violence sacrificielle qui tourne mal. ${ }^{8}$

Mais en observant plus largement le déroulement de cette tragédie dans laquelle la violence règne à tous les niveaux, il est possible d'interpréter notamment les relations conflictuelles entretenues par Héraclès et par Héra, et reflétées dans la controverse opposant Iris et Lyssa', comme la formulation d'une interrogation exprimée par Euripide sur les apories et les désastres entraînés par le règne absolu de la violence dans l'univers. En conséquence, les catégories girardiennes ont quelque chance d'être opératoires pour interpréter non pas seulement l'épisode du retournement du sacrifice, mais l'ensemble de la pièce, voire l'ensemble de ce mythe bâti sur une multitude de situations de rivalités mimétiques. D'ailleurs, en dessinant systématiquement l'image de son héros comme celle d'un bouc émissaire qui pacifie la terre et qui risque constamment sa vie pour effacer les haines nées de l'union de Zeus et d'Alcmène, ou de l'erreur sanglante commise par Amphitryon, le poète illustre toute une réflexion tragique centrée sur la notion de sacrifice ${ }^{10}$. Alors, quand à la fin de la pièce Thésée envisage la possibilité de purifier son ami Héraclès de toutes ses souillures et de le réhabiliter dans sa gloire, le poète aurait-il réussi à résoudre la « crise sacrificielle » tout en abolissant les condamnations radicales qui pèsent sur la vie d'un bouc émissaire, du fait de son statut? Mais alors quelles valeurs oppose-t-il comme remparts, face aux pulsions jalouses des hommes et à leur soif de possession et de puissance?

\section{Le déchaînement de la violence et des rivalités mimétiques dans l' Héraclès furieux}

10 Tous les détails apportés dès le prologue pour exposer la situation dramatique produisent des effets convergents, montrant de manière frappante la permanence de la violence dans l'histoire des hommes.

11 En effet, Amphitryon commence par expliquer sa présence sur le sol thébain par la nécessité d'un exil auquel l'a contraint le meurtre involontaire d'Électryon, héros de Tirynthe et père d'Alcmène ${ }^{11}$. Par ailleurs, lorsque Mégara, la femme d'Héraclès, entre en scène, elle rappelle la haute valeur guerrière de ce vieillard qui autrefois vainquit les Taphiens pour venger le meurtre des fils du même Électryon ${ }^{12}$ !

D'autre part, lorsqu'Amphitryon évoque la cité où il a trouvé refuge, il la présente comme cette «Thèbes où germa la moisson des Spartes $»^{13}$. Or, ces guerriers légendaires sont connus pour s'être entretués. Mais si, à son origine, l'histoire de la cité cadméenne est marquée par la violence, la suite de son déroulement est tout aussi troublée. En effet, au début du drame, Amphitryon, Mégara et ses enfants sont menacés de mort par cet usurpateur nommé Lycos. Ce personnage est aussi présenté comme étant le descendant 
d'un ancêtre portant le même nom, un antique maître de la cité, qui persécuta Antiope, puis qui fut tué par ses fils, Amphion et Zéthos, les Dioscures thébains ${ }^{14}$.

Des conflits privés envahissent donc en nombre la mémoire mythique. Mais les discordes civiles et les guerres étrangères ne manquent pas non plus. Ainsi, Amphitryon indique

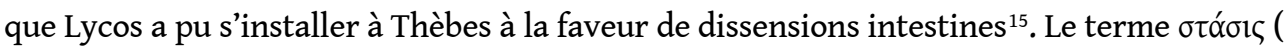
stasis) qui dénonce la crise est repris, au cours de la pièce, dans des champs sémantiques soulignant les conséquences désastreuses et les dangers mortels suscités par une telle sédition ${ }^{16}$.

Par ailleurs, Amphitryon a très vite l'occasion de rappeler les rivalités qui ont opposé, dans un passé récent, la ville de Thèbes et celle des Minyens d'Orchomène. En effet, luimême et sa famille sont installés comme suppliants auprès d'un autel dédié à Zeus Sauveur, érigé par Héraclès lors de la victoire remportée contre ces adversaires, en grande partie grâce à lui ${ }^{17}$.

Dès le début de la pièce, un véritable vertige naît donc dans l'esprit des spectateurs, confondus devant l'universalité de la violence et de la guerre, et déconcertés par le caractère inextricable, parfois jusqu'à l'absurde, des enchaînements meurtriers.

La tragédie personnelle d'Héraclès s'inscrit dans le cadre d'une violence tout aussi généralisée, n'ayant que trop tendance à se perpétuer. De fait, lorsqu'il résume l'histoire de sa vie, le héros montre tout d'abord comment il lui faut payer le prix d'actes commis avant sa naissance et demandant réparation ${ }^{18}$. Il évoque donc, notamment, la souillure contractée par Amphitryon, du fait du meurtre d'Électryon, mais surtout l'adultère commis par Zeus et Alcmène. En effet, la jalousie d'Héra le menace désormais constamment, comme fils né de cette union illégitime. La situation est clairement celle d'une "rivalité mimétique », dans laquelle une divinité féminine refuse de partager avec une mortelle l'amour de son époux ${ }^{19}$.

Par ailleurs, le héros lui-même commet des violences, tout au long de sa vie. Les actes sanglants perpétrés par Héraclès au cours de ses douze travaux relèvent quant à eux d'une catégorie particulière, puisqu'ils s'appliquent à des monstres que le héros élimine de la surface de la terre, dans une espèce de généreux rituel expiatoire. Toutefois, le poète semble indiquer qu'ils habituent, en quelque sorte, le personnage à tuer et qu'ils l'entraînent dans l'engrenage d'une autre forme de violence dont il ne perçoit pas la différence de nature :

Quelle grandeur trouvera-t-on aux combats que m'a ordonnés Eurysthée contre l'hydre et le lion, si je ne parviens pas à arracher à la mort mes propres enfants ?20

Le héros exerce alors des représailles d'ordre privé :

Quant à moi -car c'est maintenant à mon bras d'œuvrer- je pars, et tout d'abord je renverserai le palais du nouveau tyran; je trancherai sa tête impie et la jetterai en pâture aux chiens. Puis, tous les Cadméens que j'aurai trouvés ingrats envers moi qui suis leur bienfaiteur, tomberont sous les coups de cette massue victorieuse ou seront transpercés par mes flèches ailées ; j'emplirai tout l'Isménos de morts à force de carnage, et les eaux limpides de Dircé seront teintes de sang ${ }^{21}$.

19 À ce moment, Héraclès est saisi par une véritable frénésie meurtrière. Aux motifs invoqués, s'ajoute celui d'un autre type de rivalité mimétique, car, dans ces circonstances, il s'agit aussi pour lui de reconquérir le trône et le pouvoir à Thèbes. Or, sous cet aspect, la réaction du héros est présentée non seulement comme une réplique opposée à la soif de puissance manifestée par Lycos, mais aussi, fondamentalement, comme une réaction d'ambition propre, identique à celle de son adversaire. Les personnages de la tragédie 
connaissent bien le phénomène, eux qui définissent la royauté, autrefois possédée par Créon, comme :

un objet de convoitise qui provoque l'assaut des lances contre son heureux

possesseur ${ }^{22}$.

Enfin, lorsque le drame d'Héraclès se noue et lorsque, dans son délire, il assassine ses propres enfants, pensant tuer ceux d'Eurysthée, les ressorts psychologiques qu'Euripide prête à son héros apparaissent encore plus clairement comme les effets d'une compétition malsaine engagée avec cet ennemi ${ }^{23}$, car la descendance des héros est définie, dans la pièce, comme la «brillante couronne » qui brille sur leur front ${ }^{24}$. En conséquence, le meurtre des enfants constitue, sur le plan symbolique, une destitution et une prise de pouvoir. À travers cet acte de folie, Euripide représente donc un Héraclès dont se révèle le désir profond de triompher de ce rival et de ce double perçu dans Eurysthée ${ }^{25}$. En effet, l'ironie de la situation tragique fait ressortir la gémellité des deux personnages, puisque les attaques réservées à l'usurpateur s'appliquent, en réalité, au cas d'Héraclès, comme dans un système de parfaite équivalence.

21 En dernier lieu, lorsque les gestes du héros se retournent contre lui, il se retrouve dans une situation qui le transforme à nouveau en victime de la jalousie divine; il est finalement atteint par la haine d'Héra.

Le personnage d'Héraclès est donc dépeint par Euripide dans toute son ambivalence par rapport à la violence. Il la subit, en particulier comme conséquence de ses origines familiales, mais il l'exerce aussi, comme vengeance, comme acte de légitime défense pour sauver son père, sa femme et ses enfants, ou pour assurer sa gloire et son pouvoir.

23 À la fin de la pièce, le héros est vaincu, sa vie est totalement ruinée par la mort de ses enfants. La violence semble présente partout: dans le ciel de divinités vindicatives, au sein des sociétés humaines qui se font la guerre, et entre les individus qui s'affrontent. En tant que poète, Euripide constate sombrement cette diffusion universelle; manifestement, il cherche aussi une solution à la propagation sans limites de ces énergies destructrices et belliqueuses.

\section{La recherche d'une issue à la violence, dans l'Héraclès furieux}

Le tableau de la violence généralisée, en l'absence notamment de mécanisme régulateur comme celui du rituel sacrificiel, incite à imaginer l'éventualité d'une élimination complète de l'espèce humaine. R. Girard a montré que cette crainte se manifeste à travers les mythes provenant de civilisations diverses ${ }^{26}$. Dans la pièce d'Euripide, des traces de cette question apparaissent à travers la référence faite à la légende des Spartes. En effet, dans ce cas, la disparition totale de ces guerriers n'est évitée que par une mesure de sauvegarde divine qui permet la survie de cinq d'entre eux : «les survivants, sauvés en petit nombre par Arès $»^{27}$. Mais cette initiative surnaturelle répond de manière ponctuelle ou conjoncturelle aux nécessités de la situation. Elle n'établit pas les règles d'une survie harmonieuse au sein des sociétés et elle n'engage pas le comportement futur des humains. D'ailleurs, la descendance des Spartes, représentée dans la pièce sous les traits des vieux choreutes, incarne plutôt la justice et un esprit de paix. Mais le poète la montre impuissante à contrecarrer la violence importée par Lycos ou Héraclès. La solution apportée pour enrayer l'engrenage de la violence doit donc se former dans la conscience des hommes, de manière à déterminer le système politique d'une cohabitation paisible. 

d'interrompre le cycle d'une violence vengeresse en supprimant les descendants d'adversaires opprimés. Le sort des enfants est ainsi particulièrement triste et menacé, dans l'Héraclès furieux d'Euripide. Outre les difficultés propres du héros, en butte dès son plus jeune âge à la haine d'Héra, sa progéniture devient la cible de toutes les attaques, comme celle d'Eurysthée risquerait de le devenir, en cas de triomphe du protagoniste. La logique de ces assassinats visant à l'extinction de tout danger futur de représailles s'exprime dans les termes clairs d'un calcul pragmatique. Ainsi Lycos justifie sa cruauté à l'égard de la famille d'Héraclès par sa volonté « d'éteindre le meurtre par le meurtre ${ }^{28}$. Amphitryon admet, d'ailleurs, le bien-fondé de cette précaution :

Mais ces enfants pourquoi veux-tu les faire périr? Que t'ont-ils fait? Je reconnais que tu donnes une preuve de prudence, en redoutant, vil comme tu l'es, les fils d'un héros ${ }^{29}$.

Mais la portée quasiment rituelle de ces meurtres affleure sous certaines métaphores. Évidemment, la signification d'un tel sacrifice est dévoyée puisque, dans ce cas, la cérémonie n'a pas pour objectif de rétablir la paix en réconciliant des adversaires, mais le vocabulaire appliqué à l'élimination des enfants, chargé d'ironie tragique, se rapporte néanmoins au sacré et il est bien question de faire cesser une violence déréglée par le biais de cette solution inique et monstrueuse. Alors, Mégara, la mère, se lamente : « Quel est le sacrificateur, quel est l'égorgeur deces infortunés... $\|^{30}$, comme si le meurtrier était un prêtre prêt à égorger des victimes sur un autel. Toutefois, dans les mythes, la question de l'éradication de la violence n'est jamais résolue par l'élimination des dernières générations. Un vengeur survient toujours de quelque part. C'est pourquoi Amphitryon prévient Lycos :

N'use pas de violence, sinon tu subiras la violence lorsque la divinité tournera contre toi le vent de la fortune ${ }^{31}$.

Avant l'instauration de tribunaux, seul le recours au sacrifice d'une victime peut donc mettre un terme à un cycle de violence. Dans la pièce, Héraclès est présenté, à tous égards, comme la figure d'un bouc émissaire. En effet, tout d'abord, à travers l'initiative d'Héra qui cherche à faire périr le héros dans son berceau en envoyant « deux serpents aux yeux ardents $\|^{32}$, la mort qu'elle cherche à lui infliger apparaît comme la compensation qu'elle souhaiterait se voir offerte. Elle se contenterait ainsi d'éteindre la descendance d'Alcmène et de Zeus, puisqu'elle ne saurait se venger directement sur la femme aimée par le dieu souverain. Dans ce cas, Héraclès sert de substitut, dans cette vengeance dont son ennemie héréditaire attend, après ce règlement de compte, l'apaisement de sa colère. Mais l'enfant, qui est un demi-dieu, triomphe des adversaires monstrueux commandités par Héra. Pour autant, il n'échappe pas vraiment, ni de manière définitive, à la vindicte de la déesse.

En fait, cette victoire d'Héraclès transforme son statut: de victime expiatoire, il devient un bouc émissaire au sens plein du terme, c'est-à-dire que sa vie doit désormais servir à purifier la terre habitée. Chez Euripide, le sens donné au cycle des douze travaux héroïques correspond en effet à la vision d'un progrès de la civilisation autorisé par les dieux, en même temps qu'à un idéal de généreuse abnégation. À défaut d'obtenir la mort d'Héraclès, Héra semble accepter le compromis d'une existence offerte au service de la collectivité humaine et pour l'honneur des dieux. En définitive, cette solution de sacrifice purificateur est donc imposée par la déesse. Dans la pièce, l'historique des causes est formulé à plusieurs reprises, une fois par Amphitryon ${ }^{33}$, puis par Iris qui explique les 
volontés de l'épouse outragée de Zeus ${ }^{34}$, puis par Héraclès lui-même. Or, dans le dernier exposé notamment, les causalités se dessinent clairement. La déesse est alors rendue responsable de toute la série de malheurs qui constituent l'existence du héros : attaque des serpents au berceau, cycle des douze épreuves, meurtre des enfants ${ }^{35}$.

Le dernier événement advient lorsque le demi-dieu ayant triomphé de tous les monstres croit pouvoir bénéficier de la gloire qu'il a acquise du fait de ces exploits, ou tout au moins mener une vie normale au sein de sa famille. Mais dans ce cas, le sort du bouc émissaire se révèlerait profitable et avantageux. Son statut l'établirait même dans une situation de concurrence dangereuse pour les dieux. Iris explique d'ailleurs ainsi l'exigence de destruction du héros imposée par Héra:

Tant qu'il n'avait pas achevé ses durs travaux, le destin le protégeait et Zeus le père ne permettait jamais à Héra ni à moi de lui faire du mal. Maintenant qu'il est arrivé au terme des épreuves imposées par Eurysthée, Héra veut qu'il se souille du sang des siens par le meurtre de ses enfants [...]. Les dieux compteront pour rien et la race mortelle aura la puissance, si Héraclès n'est point puni. ${ }^{36}$

Effectivement, au terme de ses travaux, Héraclès a appris les secrets des Enfers et il a triomphé des puissances de la mort, en particulier en leur arrachant son ami Thésée ${ }^{37}$. Le bouc émissaire, chargé des souillures qu'il expie, ne peut donc pas prétendre à une réhabilitation sans remettre en cause, de manière très subversive, la souveraineté des dieux. Sa qualité de victime sacrificielle lui est donc acquise pour toujours. Il lui est interdit de reconstruire sa vie.

31 La mort des enfants d'Héraclès s'explique ainsi. En effet, d'après la pensée illustrée dans la pièce, la gloire humaine, pour être accomplie, doit se prolonger dans le bonheur de sa postérité ${ }^{38}$. En revanche, le héros, privé de descendance, n'aura plus vraiment d'existence reconnue. Il ne peut en être autrement dans le cas d'un bouc émissaire, être sacré qui délivre l'univers de ses fléaux, mais qui paie de son anéantissement social, sinon de sa vie, la grandeur de son image ${ }^{39}$.

La tragédie met en lumière le caractère poignant du sort dévolu aux boucs émissaires. Chez Sophocle, le destin d'œEdipe, tel que l'évoquent notamment J.-P. Vernant et P. VidalNaquet, apparaît comme fascinant, pitoyable et terrible. Dans l'œuvre d'Euripide, de plus, les personnages contestent le bien-fondé d'une telle pratique de purification des sociétés qui, au nom du sacré, admet le sacrifice d'êtres innocents et détruit l'idée de justice.

Mais, comment supprimer cette catégorie religieuse du bouc émissaire dans une perspective où elle n'est pas même remplacée par l'établissement d'un système juridique et de tribunaux ? Comment réussir ainsi à lutter contre l'extension de la violence ? Dans les termes de la démonstration anthropologique élaborée par R. Girard, la solution est difficile à trouver dans une société païenne, ou plutôt non chrétienne. En effet, dans sa vision de l'histoire des hommes, le don du Christ, réalisé une fois pour toutes, peut seul réussir à abolir toute nécessité sacrificielle. Alors quand les héros grecs civilisateurs, personnages d'Euripide, Héraclès ou Thésée, se révoltent contre la victimisation tyrannique des boucs émissaires, que peuvent opposer à la logique religieuse la morale et la spiritualité antiques?

\section{La réhabilitation d'Héraclès, bouc émissaire}

En effet, telle est la problématique explorée par Euripide qui recompose et réinterprète les données mythiques en fonction des valeurs d'une éthique du dévouement et, en 
quelque sorte, d'une mystique des solidarités humaines, plutôt que de suivre les codes d'échange expiatoires de la religion traditionnelle.

De fait, le but du poète consiste à substituer aux règles, aux coutumes, aux croyances des cultes organisés par la société grecque, les motivations et les exigences d'une conscience humaine créatrice de sa morale et innovant dans la foi. Autrement, l'introduction d'un double système de causalité expliquant la prise en charge par Héraclès de l'accomplissement des douze travaux tantôt comme une nécessité imposée par les dieux, tantôt comme un devoir que le héros choisit de s'imputer ne se justifierait pas. Or, même si Amphitryon hésite à formuler une hypothèse complètement dégagée de ses fondements fatalistes et religieux, néanmoins il présente avant tout la geste d'Héraclès comme une initiative correspondant à un idéal de générosité :

il a cherché à adoucir mon infortune et à obtenir notre rentrée dans la patrie en offrant à Eurysthée - prix bien haut pour notre retour - de purger la terre de ses monstres : c'était peut-être Héra qui, le maîtrisant de son aiguillon, le forçait à cette tâche, peut-être était-ce simplement l'arrêt de la destinée..$^{40}$

Quant au héros, il revendique la reconnaissance du courage manifesté au cours de ses épreuves endurées comme pour obéir à une discipline intérieure :

Innombrables aussi sont les épreuves dont j'ai goûté ; je n'en ai refusé aucune ${ }^{41}$.

37 En mettant ainsi en relief la psychologie de son personnage, Euripide favorise l'expression d'un jugement personnel appréciant de manière critique le sens et le bienfondé des pratiques rituelles, voire inventant une piété nouvelle.

Car Héraclès ne croit plus aux vertus d'un commerce entretenu avec les dieux qui achèterait leurs faveurs et la paix restaurée entre les hommes au prix de la souffrance endurée par une victime innocente. Mais il remplace cette vision traditionnelle par une théologie beaucoup plus épurée dont les principes énoncent la complétude et la perfection des dieux :

la pensée que les dieux s'adonnent à des amours coupables ne peut être la mienne, pas plus que je n'ai jamais admis et que je ne croirai jamais qu'ils chargent mutuellement leurs bras de chaînes, ni que l'un commande en maître à l'autre ; les récits contraires sont de misérables inventions des poètes. ${ }^{42}$

Le décalage qui s'établit entre le cadre mythologique et ces réflexions insérées dans le drame est frappant. Euripide révèle ainsi comment les questions obsédant une conscience animée par le sentiment du tragique bouleversent la quiétude des idées religieuses acquises. En l'occurrence, si Héraclès apparaît comme le théoricien d'une pensée nouvelle, Thésée qui l'accompagne dans cette aventure de l'esprit met en application un comportement révolutionnaire, vis-à-vis d'un être porteur des souillures d'une violence sanglante et, spécialement, du bouc émissaire que représente Héraclès.

En effet, il se déclare prêt à l'accueillir dans la cité athénienne, à lui procurer des ressources et à le réhabiliter :

Ce sera une belle couronne, pour notre cité, que la gloire obtenue dans toute la

Grèce en servant un grand homme. ${ }^{43}$

Or, d'après les conceptions religieuses traditionnelles, les miasmes qui contaminent un meurtrier (ou un bouc émissaire) le rendent impur de sorte qu'il ne peut être touché par quiconque, de sorte aussi qu'il ne peut pas paraître librement à la face du soleil sous peine de répandre la souillure dans l'univers des hommes et des dieux. Interdit de séjour partout où il passe, un tel être est donc exclu du monde des vivants. Mais Thésée triomphe complètement des craintes inspirées par cette conception du sacré. Ses gestes 
sont d'une hardiesse inouie : il se propose, en effet, de purifier lui-même les mains de son ami sans redouter un contact néfaste ${ }^{44}$ et il proclame lui aussi sa conviction d'une nature inaltérable des dieux, les mettant à l'abri de toute atteinte impure :

L'être mortel ne peut souiller rien de divin. ${ }^{45}$

En conséquence, l'accomplissement d'un rituel de purification n'apparait plus comme la réparation d'une offense blessant directement les divinités, mais plutôt comme la manifestation de la volonté du fautif, conscient de son erreur, qui cherche à se réinsérer dans la communauté humaine, dans l'intention de ne plus jamais s'attaquer à l'harmonie du monde et de ne plus faillir au respect dû à la vie. L'acte religieux de purification exprime alors une démarche de renonciation à la violence. Tel est précisément le parti librement adopté par Héraclès, après mûre réflexion, à la fin de la pièce. En fait, le déroulement dramatique conduit le personnage de l'extermination civilisatrice des monstres à la conquête brutale d'un pouvoir personnel, puis à l'accident du meurtre de ses enfants pour aboutir à la recherche des conditions d'un état de non violence. Car, Euripide semble faire suivre à son héros un cheminement propice à lui faire prendre conscience de la totale abnégation convenant à sa figure de purificateur universel et de champion de la paix. De fait, à la fin de la pièce, la notion de souillure n'existe plus autrement que comme l'idée d'un grave manquement commis envers l'existence d'autrui, et la violence est bien près d'être éradiquée.

Le débat qu'Héraclès engage avec lui-même, au moment de décider s'il convient pour lui de partir en exil en gardant ou en abandonnant ses armes meurtrières de sa femme et de ses enfants, constitue un signe éloquent de la prise de conscience du héros et de sa volonté d'éviter désormais toute violence offensive et agressive ${ }^{46}$. En effet, si le personnage juge préférable, en dernier lieu, de ne pas s'avancer désarmé dans l'avenir désolé qui se présente à lui, il justifie le parti qu'il prend par son souci de «résister à la tentation de la mort $»^{47}$. Car s'engager sans les instruments de ses glorieux exploits dans un monde qui demeure déchiré et hostile équivaut à courir le risque mortel d'une attaque ennemie, selon une attitude suicidaire. Or, Héraclès repousse l'idée d'une telle extrémité qui lui apparaît comme la marque d'une lâcheté, d'une fuite face à la dureté du destin ${ }^{48}$. Mais Euripide apporte un soin particulier à définir la fonction dissuasive et strictement défensive dévolue à ces armes qui ont fait la gloire et la ruine du héros. En effet, son personnage requiert la présence de Thésée à ses côtés, lorsqu'il ira clore auprès d'Eurysthée le temps des épreuves et le cycle des douze travaux :

Thésée, il est un service que je te demande : viens à Argos afin d'y régler avec moi le prix qui m'est dû pour avoir ramené le chien des Enfers : si j'y vais seul, je crains que ma douleur de n'avoir plus d'enfants ne cause un malheur. ${ }^{49}$

Il exprime alors sa crainte de se laisser à nouveau emporter par la violence, comme l'imaginent, par ailleurs, non sans quelque vraisemblance, les auteurs d'autres versions de la légende. Mais, pour sa part, le poète tragique dessine désormais le portrait d'un héros résolu à ne plus laisser la haine se développer en lui, même à l'égard d'adversaires, d'ennemis dont la chance d'avoir des enfants vivants fait, en outre, des rivaux trop heureux.

À l'issue de cette tragédie dans laquelle Euripide a déployé toutes les ressources de son art pour mettre en scène les mécanismes de la propagation incontrôlable d'une violence déréglée, le protagoniste fixe donc sa conduite en énonçant les règles d'une non violence fragile et toujours menacée par les pulsions d'agressivité naturelle qui se déclenchent inévitablement, face à l'arrogance d'un concurrent avantagé par le sort. Mais Héraclès 
connaît un remède puissant pour résister victorieusement à la tentation de la violence. En effet, il attend de Thésée l'assistance efficace que peut apporter un compagnon ayant partagé la découverte des espaces réservés à Hadès et l'aventure d'un retour solidaire des Enfers ${ }^{50}$.

En effet, cette expérience est d'ordre initiatique. Euripide le signale clairement, en passant, lorsque son personnage explique à Amphitryon qui l'interroge sur l'effectivité incroyable de ce voyage chez les divinités souterraines que, pour réussir à revenir du monde des morts, il a dû apprendre à connaître les mystères ${ }^{51}$. L'exploit consistant à en ramener une autre personne, Thésée en l'occurrence, constitue un événement tout à fait exceptionnel dans la mythologie grecque ${ }^{52}$. Sur le plan symbolique, en revanche, dans l'enseignement des mystères d'Éleusis, l'amitié et le partage d'un compagnon élu pour la vie permettent d'échapper aux épreuves de l'existence et à la mort ${ }^{53}$. Dans toute la pièce, la recherche de l'aide apportée par un ami revient comme une préoccupation lancinante. Manifestement, compte tenu du déroulement dramatique et du rôle joué par Thésée s'acquittant de sa dette de reconnaissance envers Héraclès, le poète indique qu'à ses yeux, cette quête permet d'abolir les engrenages de violence destructrice qui menacent la condition humaine ${ }^{54}$.

47 Sur le plan dramatique, Euripide propose donc l'idéal rayonnant de la civilisation athénienne, incarné par le mythique roi Thésée, contre les brutalités sauvages, obscures de Thèbes ou d'Argos. À une image primitive des systèmes de régulation de la violence fondés sur l'élection d'un bouc émissaire, personnage sacré et horrifique, définitivement exclu et sacrifié sans état d'âme par la société dont il rétablit les équilibres au prix de son effacement du monde des humains, elle substitue donc une éthique de la paix et de la solidarité, autorisant ainsi la purification de l'être humain, quelle que soit la gravité de son erreur, devant des divinités ennoblies par une théologie issue d'une vraie spiritualité. La spiritualité d'Éleusis contre les mécanismes de rivalité mimétique... Le poète suggère aussi cette solution, recommandant la mise en œuvre d'une exigence humaine, d'une éthique de la générosité, de l'abnégation, de la reconnaissance et de l'amitié, représentées successivement par Héraclès et par Thésée, contre l'automatisme des instincts de possession.

Le système est sans doute aussi précaire que la foi placée par Héraclès dans ses propres capacités de tempérance et de modération, lorsqu'il s'agit de fréquenter un tyran comme Eurysthée et de parlementer avec lui. Néanmoins, à travers sa tragédie, Euripide illustre bien une pensée qui cherche à éliminer l'agressivité et la violence, y compris dans une perspective religieuse où les pratiques de purification, sinon de sacrifice, conservent leur valeur. Le drame d'Héraclès se situe donc au-delà du moment dit de "crise sacrificielle " dans la théorie de R. Girard. Il se place en effet à un stade de la pensée où le bien-fondé des rituels est assuré non plus par une logique comptable d'expiation, abolie par les critiques suscitées par un esprit de justice, mais par la piété épurée des mystères, par l'exercice de la conscience morale et par le développement du sens de l'humain. 


\section{NOTES}

1. La violence et le sacré (= V. S.), Paris, Hachette, 1972 ; les références données dans cet article renvoient à la pagination de l'ouvrage réédité chez Hachette Littérature, 2002, Collection Pluriel (Philosophie). Pour Des choses cachées depuis la fondation du monde (Paris, Grasset, 1978), les références renvoient à la pagination de l'ouvrage dans la réédition de la Librairie Générale Française, 2006, coll. Le Livre de Poche, Collection Biblio Essais.

2. Héraclès furieux, v. 922 sqq.

3. Sur le mythe d'Héraclès, cf. O. Gruppe, RE, Suppl ${ }^{t}$ Band III, 1918, s. v. « Herakles ", col. 1018 et G. Karl Galinsky, The Herakles Theme, Oxford, Blackwell, 1972 ; sur l'organisation originale des données du mythe, cf. U. von Wilamowitz-Mollendorff, Euripides. Herakles, Darmstadt, Wissenschaftliche Buchgesellschaft,1959, II, p. 51 sqq., G. Karl Galinsky, The Herakles Theme, p. 58 et Godfrey W. Bond, Euripides. Heracles, Oxford, Clarendon Press, 1981, p. XXVIII-XXX.

4. V.S., p. 65.

5. V.S., p. 67.

6. Cf. Héraclès furieux, v. 16 sqq. ; 1256 sqq.

7. V. S., p. 67.

8. V.S., p. 64.

9. Héraclès furieux, v. 822-874.

10. Sur le bouc émissaire et sa fonction purificatrice dans les rituels grecs, cf. J.-P. Vernant et P. Vidal-Naquet, Mythe et tragédie en Grèce ancienne, Paris, Maspero, 1981, p. 124. En rapport avec la tragédie d'Euripide, l'expression indique une fonction dramatique et sociale, plutôt qu'un statut sacré, au sens propre du terme.

11. Héraclès furieux, v. 16-17. Sur cet épisode de la légende, cf. Hésiode, Le Bouclier, v. 82, 86.

12. Héraclès furieux, v. 61. Cf. Hésiode, Le Bouclier, v. 1 sqq.

13. Cf. Héraclès furieux, v. 4-6. Les traductions des passages cités d'Héraclès sont personnelles.

14. Héraclès furieux, v. $26 \mathrm{sqq}$.

15. Héraclès furieux, v. 34 .

16. Cf. Héraclès furieux, v. 588 sqq.

17. Cf. Héraclès furieux, v. 48-50. G. W. Bond note qu'Orchomène fut la rivale traditionnelle de Thèbes pendant plusieurs siècles de son histoire (Euripides. Heracles, p. 74).

18. Cf. Héraclès furieux, v. 1256 sqq. Héraclès remontant aux origines de son malheur qui ont précédé sa naissance, procède comme ๔Edipe, autre personnage de la mythologie grecque qui résout la malédiction héréditaire accablant sa famille en remplissant la fonction de bouc émissaire (cf. Euripide, Phéniciennes, v. 1597 sqq.).

19. Dans le même registre, ailleurs dans la pièce, Amphitryon reproche à Zeus de l'avoir contraint à un partage illégitime en prétendant à l'amour d'Alcmène: "Toi, tu as su entrer secrètement dans mon lit et t'emparer sans autorisation de l'épouse d'un autre » (Héraclès furieux, v. 344-345).

20. Héraclès furieux, v. 578-581.

21. Héraclès furieux, v. 565 sqq.

22. Héraclès furieux, v. 65-66. Cf. G W. Bond : "Tyranny, power, and wealth are subject to envy » ( Euripides. Heracles, p. 81). L'idée est un lieu commun dans la littérature grecque.

23. Héraclès furieux, v. 982 sqq. : «Voilà, mort, un des rejetons d'Eurysthée ; maintenant qu'il est tombé, il m'a vengé de la haine de son père. »

24. Cf. Héraclès furieux, v. 839. 
25. Cf. R. Girard : «Le "destin“, toujours ironique - il ne fait qu'un avec la violence - fait accomplir à Héraclès le projet sinistre de son rival ; c'est lui, en fin de compte, qui sacrifie sa propre famille. Plus la rivalité tragique se prolonge plus elle favorise la mimesis violente, plus elle multiplie les effets de miroir entre les adversaires » (V.S., p. 75). Il évoque, en l'occurrence, Lycos, comme rival, mais tous les rivaux sont des doubles.

26. Cf. Des choses cachées..., p. 140 sqq.

27. Héraclès furieux, v. 6.

28. Héraclès furieux, v. 40.

29. Héraclès furieux, v. 206 sqq.

30. Héraclès furieux, v. 451. Sur l'ironie tragique de ce vocabulaire métaphorique, cf. G. W. Bond, Euripides. Heracles, p. 180.

31. Héraclès furieux, v. 215-216.

32. Héraclès furieux, v. 1266 sqq.

33. Héraclès furieux, v. 16 sqq.

34. Héraclès furieux, v. 827 sqq.

35. Héraclès furieux, v. 1263 sqq.

36. Héraclès furieux, v. 827-832; 841-842.

37. Héraclès furieux, v. 610 sqq.

38. Héraclès furieux, v. $67,885-886$, etc.

39. Sur le statut et la figure du bouc émissaire, cf. J.-P. Vernant et P. Vidal-Naquet, Mythe et tragédie en Grèce ancienne, Paris, Maspero, 1972, p. 99 sqq. (chapitre: "Ambiguïté et renversement. Sur la structure énigmatique d'(Edipe-Roi »).

40. Héraclès furieux, v. 16 sqq.

41. Héraclès furieux, v. 1353-1354.

42. Héraclès furieux, v. 1341-13446.

43. Héraclès furieux, v. 1334-1335.

44. Héraclès furieux, v. 1324.

45. Héraclès furieux, v. 1232.

46. Héraclès furieux, v. 1378.

47. Héraclès furieux, v. 1351.

48. Sur cette attitude d'Héraclès, opposée à celle de l'Ajax de Sophocle, cf. A. Garzya, Pensiero e tecnica drammatica in Euripide, Naples, Libreria Scientifica Editrice, 1962, p. 28 ; J. de Romilly, La modernité d'Euripide, Paris, P. U. F., 1986, p. 69 et « Le refus du suicide dans l'Héraclès d'Euripide », Archaiognôsia, I, 1980, p. 1-10 et J. Assaël, Euripide, philosophe et poète tragique, Namur, Peeters, 2001, p. 179.

49. Héraclès furieux, v. 1386-1388. L. Parmentier précise que «le prix est le retour à Argos ; cf. v. 18-25. Héraclès craint de s'emporter contre Eurysthée et de se venger de lui. D'après une version, il avait tué les trois fils d'Eurysthée (Athénée IV 157 F). Si ce passage manquait, rien ne nous avertirait d'une lacune, et il donne un détail introduit ici pour une raison que nous ignorons » ( Euripide. Héraclès, Paris, C. U. F., [1923] 1976, p. 73, n. 1). Si, sur le plan dramatique, l'indication ajoutée par Euripide apparaît comme inutile, en revanche elle est fort intéressante sur le plan de l'établissement d'une philosophie de la vie.

50. Sur l'aspect déterminant de cette épreuve, cf. J. Assaël, «L'Héraclès d'Euripide et les ténèbres infernales ", Les Études Classiques, 62, 1994, p. 318.

51. V. 613. Sur l'aspect initiatique du mythe, cf. F. Bader, "Sémiologie des travaux d'Héraclès », in Visages du destin dans les mythologies. Actes du colloque de Chantilly [ $\left.1^{\mathrm{er}}-2 \mathrm{mai} 1980\right]$, Paris, 1983, p. 61 sqq.

52. Cf. J. Assaël, « La résurrection d'Alceste », R. E. G., 117, Janvier-juin 2004, p. 37-58.

53. Cf. V. Magnien, Les mystères d'Éleusis, Paris, Payot, 1929, p. 187. 
54. Sur le pacifisme de la religion d'Éleusis, cf. S. S. M. De Carvalho, «Les mystères d'Éleusis ", Dialogues d'Histoire Ancienne, 18/2, 1992, p. 93-135.

\section{RÉSUMÉS}

René Girard interprète la scène de l'Héraclès furieux d'Euripide dans laquelle échoue un rituel de purification accompli par le héros après le meurtre du tyran Lycos, comme l'illustration d'une «crise sacrificielle», selon la terminologie qu'il adopte notamment dans La violence et le sacré. En fait, ce type de lecture girardienne peut s'appliquer à l'ensemble de la pièce et il est alors possible de discerner la recherche menée par le poète tragique pour trouver des solutions capables d'enrayer les mécanismes de la violence propres à la nature humaine.

Rene Girard interprets the scene of the Heracles of Euripides in which failed a ritual purification performed by the hero after the murder of the tyrant Lycos, as the illustration of a "sacrificial crisis," according to the terminology he adopts in his book: La violence et le sacré. In fact, this type of reading may be applied to the entire play and it is then possible to discern the research conducted by the tragic poet to find solutions that could stop the mechanisms of violence inherent in the human nature.

\section{INDEX}

Mots-clés : Euripide, Héraclès furieux, sacrifice, souillure, violence

Keywords : Euripides, Herakles, pollution, sacrifice, violence

\section{AUTEUR}

\section{JACQUELINE ASSAËL}

Professeur de langue et littérature grecques à l'Université de Nice-Sophia Antipolis. Membre du Centre Transdisciplinaire d'Épistémologie de la Littérature. EA 1758 / UNSA.Auteur de: Intellectualité et théâtralité dans l'oeuvre d'Euripide, Nice, 1993. (Publications de la Faculté des Lettres et Sciences Humaines de Nice) ; Euripide, philosophe et poète tragique, Namur/Louvain, éd. Peeters, Coll. d'Etudes Classiques, $n^{\circ} 16,2001$; Pour une poétique de l'inspiration, d'Homère à Euripide, Namur/ Louvain, éd. Peeters, Coll. d'Etudes Classiques, n² 21, 2006. 